\title{
Fisher's Fundamental Theorem of Natural Selection Isn't Fundamental After All
}

\author{
Salvador Cordova
}

DOI: 10.33014 /issn.2640-5652.2.2.cordova.1

\section{Abstract}

Fisher's Fundamental Theorem of Natural Selection (FTNS) was called "biology's central theorem" (Fisher, 1930, pgs. 36-37; Brockman, 2011; Royal Society, 2020). FTNS might possibly have been accorded this status for decades because Fisher himself declared his own theorem to be fundamental to biology (Fisher, 1930, pgs. 36-37). However, the idea that Fisher's theorem is biology's central theorem is by-and-large a myth promoted by popular science writers like Richard Dawkins (Brockman, 2011). Joseph Felsenstein, when delivering the 2018 Fisher Memorial Lecture declared that FTNS was "alas, not so fundamental" (Felsenstein, 2018; Felsenstein, 2017, pg. 94). One may be hard-pressed to find a biology textbook or biology student who can explain how FTNS helps them understand biology. Even the meaning and proof of the FTNS have remained contentious even to this day (Price, 1972; Basener and Sanford, 2018).

Not only does FTNS do little to nothing to explain biological evolution, but like most population genetic and evolutionary literature, FTNS relies on a definition of fitness in terms of population growth rates rather than the biophysical notions of fitness which are more in line with the common-sense intuitions of the medical and engineering communities.

From the perspective of the biophysical (rather than the population growth) notion of fitness, natural selection might be more accurately described as an agent against the increase of complexity rather than an agent for it. Thus, metaphorically speaking, some sort of anti-Weasel model of natural selection might better describe how selection actually works in nature rather than Dawkins' Weasel or other man-made genetic algorithms.

However, the main focus of this article is to provide some pedagogical insights through simple numerical illustrations of Fisher's theorem. The hope is that this will show the general irrelevance of FTNS to the question of the evolution of complexity by means of natural selection, and thus show that Fisher's theorem is not so fundamental after all.

\section{Introduction: The Problem of Defining Fitness}

In order to understand the meaning of Fisher's Fundamental Theorem of Natural Selection, it is helpful to understand the definition of fitness which Fisher was using. This is not so trivial a problem because in a 2009 paper on fitness, Allen Orr quipped, "biologists have offered a staggering number of definitions of fitness" (Orr, 2009), and worse Richard Lewontin lamented, "it is not entirely clear what fitness is" (Lewontin, 2003).

Andreas Wagner went even further:

However, fitness is hard to define rigorously and even more difficult to measure... An examination of fitness and its robustness alone would thus not yield much insight into the opening questions. Instead, it is necessary to analyze, on all levels of organization, the systems that constitute an organism, and that sustain its life. I define such systems loosely as assemblies of parts that carry out well-defined biological functions.

(Wagner, 2005, pg. 1)

Ironically, "systems loosely as assemblies of parts that carry out well-defined biological functions" sounds remarkably similar to Michael Behe's definition of Irreducible Complexity: "A single system composed of several well-matched, interacting parts that contribute to the basic function of the system" (Behe, 1996, pgs. 39-40).

The population-genetic/evolutionary biology definition of fitness seems disconnected, if not offensive, to common sense notions of fitness used by the medical and engineering community. For example, in the medical sense, sickle cell anemia is a heritable trait that can lead to sickness, but it is viewed as a "beneficial" adaptation in the populationgenetic/evolutionary sense. Natural selection could selectively favor loss of optical structure in Gammarus minus (an aquatic creature that dwells in caves) or tusks in Elephants. Allen Orr used the example of Gammarus minus 
to chide Daniel Dennett's naïve views of natural selection when Orr pointed out:

In reality, selection "sees" only brute birth, death, and reproduction, and knows nothing of Design. Selection-sheer, cold demographics - is just as happy to lay waste to the kind of Design we associate with engineering as to build it. Consider the eyes of cave organisms who live in total darkness. If eyes are expensive to make, selection can wreck their exquisite engineering just as surely as it built it. An optic nerve with little or no eye is most assuredly not the sort of design one expects on an engineer's blueprint, but we find it in Gammarus minus. Whether or not this kind of evolution is common, it betrays the fundamental error in thinking of selection as trading in the currency of Design.

(Orr, 1996)

In fact "reductive evolution" (loss of genes and function) rather than "constructive evolution" appears to be the dominant mode of evolution throughout most of geological history (Wolf and Koonin, 2013), but punctuated with episodes of complexification that have no clear mechanistic explanation. Reductive evolution has also been confirmed by direct observation in the field and in the laboratory to be the dominant mode of evolution (Behe, 2010). This is in contrast to a widespread and long-standing historic and present belief that natural selection generally leads to increase in complexity, and the basis for these beliefs not rooted in empiricism but either conjecture or computer simulation far removed from actual experiments (Darwin, 1859; Adami, Ofria, and Collier, 2000; Vaughan, 2019).

Amusingly, the paucity of direct examples of complexity increase and the abundance of complexity decrease by natural selection was unwittingly highlighted by Sharon Moalem's book, Survival of the Sickest, where he argues why we need birth defects like Tay-Sach's disease on account of natural selection (Moalem, 2008). Furthermore, Moalem went on to celebrate the facts of disease as evidence of Darwin's genius.

There are a variety of the notions of the term, "fit," and the population genetic/evolutionary definition (elaborated below) seems idiosyncratic relative to historical and common notions of the word. An aircraft is "fit" to fly, a submarine is fit to operate underwater, a car is fit to operate on land, a spaceship in space, etc. None of these notions of fit need be conflated with reproductive success. Biophysicists have established eyes are fit to sense and count individual photons (Bialek, 2015), that bacteria can count individual molecules (Bialek, 2012), that sharks are fit to sense electric fields in conditions at the extreme limit permissible by physics (Angier, 2010), and birds are fit to fly and navigate thousands of miles via compasses that leverage quantum spin chemistry (Ritz, Adem, and Schulten, 2000; Rubenstein and Hack, 2013).

Although the definition and treatment of fitness in the population-genetic/evolutionary sense may seem irrelevant if not repugnant to common sense notions of fitness in the medical and engineering sense, one cannot understand Fisher's theorem without understanding the populationgenetic/evolutionary definition of fitness. Therefore, the next section will be devoted to elucidating the populationgenetic/evolutionary definition of fitness. The stated definitions follow from conventions used in Joseph Felsenstein's graduate-level textbook, Evolutionary Theoretical Genetics (Felsenstein, 2017).

The population-genetic definition of fitness arises in the context of a highly idealized model that approximates some real world populations in an exponential growth stage. The notion of fitness in this simplest of models can be extended, with a modest amount of amendment, to definitions used by a large amount of population genetics literature.

However, the staggering amount population genetics literature that uses such simplistic definitions does not at all imply these population genetic models are accurate or useful. Felsenstein points out:

Fisher's and Wright's one-locus equations turn out to be approximations, sometimes bad ones... the mathematical tools at hand have not revolutionized our understanding of the evolutionary process. . many evolutionists will fail to find the clear and simple messages that population genetics theory once seemed to promise.

(Felsenstein, 2017)

Unfortunately, many preliminaries are needed to provide a cursory understanding of the terminology associated with the population-genetic/evolutionary definitions of fitness required to understand Fisher's theorem. One must suffer through some of the math in order to understand what Fisher's theorem means. 


\section{Absolute "Darwinian" Fitness vs. Relative Fitness}

Because populations can grow exponentially, it is possible that a single molecular change in one organism can result in immense macroscopic consequences for the population. For example, in the evolution of bacteria or viruses, the emergence of a novel mutational change in their genomes can result in their epidemic spread which has very noticeable effects on the macro scale. In light of the exponential growth of such populations, there is reasonable justification for choosing an exponential function for modeling growth of populations such as these, and this leads to the beginning of a mathematical definition of fitness in the sense of population genetics.

The exponential growth function arises out of one of the most elementary differential equations:

$$
x(t)=\frac{\mathrm{d} x(t)}{\mathrm{d} t}
$$

Where $x(t)$ is the size of the population and is a function of time. One of the simple solutions to this differential equation is:

$$
x(t)=A e^{t}
$$

where $A$ is a constant.

A slightly more complex differential equation that is traditionally related to population growth is

$$
\alpha x(t)=\frac{\mathrm{d} x(t)}{\mathrm{d} t}
$$

where $\alpha$ is a constant.

A solution for $x(t)$ under the constraint of (3) is:

$$
x(t)=A e^{\alpha t}
$$

Where $A$ again is also a constant. $\alpha$ is customarily called the continuous growth constant and $A$ the initial population size. This equation can serve as an idealization of exponentially growing populations.
Note that for $t=0$,

$$
x(0)=A
$$

A discrete version of (4) can be synthesized by defining the following constant which we'll call the absolute fitness or Darwinian fitness:

$$
W \equiv e^{\alpha}
$$

[It bears mentioning that calling $W$ "Darwinian fitness" might be a misnomer, since Lewontin argues implicitly that describing fitness in this way violates the spirit of what Darwin meant by fitness. Lewontin said, "Darwin's sense of fit has been bypassed" by using reproductive schedules themselves rather than the functional aspects of an organism in relation to its environment (Lewontin, 2003)].

We can then define the size of the population $P(k)$ as a function of the number of generations

$$
P(k) \equiv P(0) W^{k}
$$

where $k$ is a non-negative integer representing a particular generation cycle of the population, and $P(0)$ is the initial size of the population in generation zero. The assumption is that in such a population, all parents have their children simultaneously at the same time, and $W$ represents the average number of children each parent has, and for simplicity it is assumed $W$ is constant for all time.

If we restrict the values of $t$ to be only discrete points such that $t=k$, and noting that $P(k)$ is defined in terms of $W^{k}$, we can relate $P(k)$ to the continuous time version of population size $x(t)$. In other words, if we assume $t$ has integer values and that the units of time are stated in generations rather than seconds or years, so that $t=1$ corresponds to the amount of time for one generation, $t=2$ is the amount of time for two generations, etc., then under these constraints we can then make this relation:

$$
W^{k}=\left\{e^{\alpha}\right\}^{k}=\left\{e^{\alpha}\right\}^{t}=e^{\alpha t} .
$$

If further we let the constant $P(0)=A$, then

$$
P(0) W^{k}=A e^{\alpha t}
$$

Again, there is an implicit assumption laid out so far; 
namely every parent of generation $\mathrm{k}$ will bear all their offspring for generation $\mathrm{k}+1$ simultaneously.

If we have a population composed of $N$ sub populations that each obey the above simple model, we can extend (7) to cover multiple sub populations. With $i \in\{1,2, \ldots N\}$, the size $P_{i}(k)$ of each the $i$ sub populations in generation $k$ is:

$$
P_{i}(k)=P_{i}(0) W_{i}^{k}
$$

Note that capital " $P$ " is used to designate absolute population numbers. Depending on the context, $P_{i}$ can describe individuals and/or alleles. For the sake of simplicity, we will discuss the special case where the number of individuals is the same as the number of alleles, namely a haploid asexual population where only one gene locus is considered. Aspects of this simple special case will not hold true for diploid populations but is used primarily for pedagogical purposes.

The total population $P_{\text {tot }}$ for a given generation is straight forwardly stated as the sum of the sizes of the individual $i$ sub populations:

$$
P_{\text {tot }}(k)=\sum_{i=1}^{N} P_{i}(k)
$$

The proportion, the relative frequency, or simply frequency $p_{i}(k)$ (lower case $\mathrm{p}$ ), of alleles/individuals of a given subpopulation $i$ that appear in the total population in generation $\mathrm{k}$ is:

$$
p_{i}(k)=\frac{P_{i}(k)}{P_{\text {tot }}(k)}
$$

$p_{i}(k)$ is often called the gene frequency, although strictly speaking it is more properly called the allele frequency.

In a somewhat analogous manner, instead of absolute fitness $W_{i}$ of each of the individuals of a sub population, we can state the relative fitness $w_{i}$ of each of the individuals. Because absolute fitness is designated with a capital $W$, it is customary to designate relative fitness with a lowercase $w$.

A method of defining relative fitness, though not the only way, is to take the highest absolute fitness value found in a total population and designate it as the reference absolute fitness $W_{\text {ref. }}$ We can then define relative fitness $w_{i}$ for each subpopulation in relation to $W_{\text {ref }}$ as follows:

$$
w_{i} \equiv \frac{W_{i}}{W_{\text {ref }}}
$$

For example, if

\begin{tabular}{|l|l|}
\hline$W_{1}$ & 4.0 \\
\hline$W_{2}$ & 3.0 \\
\hline$W_{3}$ & 2.0 \\
\hline
\end{tabular}

then $W_{\text {ref }}=4.0$, thus:

\begin{tabular}{|l|c|}
\hline$w_{1}$ & 1.0 \\
\hline$w_{2}$ & 0.75 \\
\hline$w_{3}$ & 0.50 \\
\hline
\end{tabular}

\section{Mean and Variance of Relative Fitnesses}

For an arbitrary generation $k$, we can define a mean of the relative fitnesses $\overline{w(k)}$ as:

$$
\overline{w(k)} \equiv \sum_{i=1}^{N} p_{i}(k) w_{i}
$$

We can also define the variance of relative fitnesses $\operatorname{Var}(w(k))$ for a given generation $k$ as:

$$
\operatorname{Var}(w(k)) \equiv \sum_{i=1}^{N}\left\{p_{i}(k) w_{i}^{2}\right\}-\overline{w(k)}^{2}
$$

Also let us define the change of mean relative fitnesses $\Delta \overline{w(k)}$ as:

$$
\Delta \overline{w(k)} \equiv \overline{w(k+1)}-\overline{w(k)}
$$

This definition of $\Delta \overline{w(k)}$ may or may not be in agreement with other texts, but it will nevertheless be sufficient to derive a proof of Fisher's Fundamental Theorem of Natural Selection in the discrete generation single locus haploid case.

When it is said, "the fitness of the population is increasing," it usually means $\overline{w(k)}$ is increasing. Qualitatively, it

\section{Communications Blyth Institute}


simply means the individuals with the highest reproductive rate are occupying a larger and larger proportion of the population. If there is only 1 type of individual with the highest fitness, a fitness increase means that the population is asymptotically approaching a state of being homogeneous.

With these concepts now defined, we can now examine some numerical examples that give insights into Fisher's Fundamental Theorem of Natural Selection.

\section{Numerical Examples to Illustrate Fisher's Theorem}

Repeating the example in Section 2, suppose we have the following scenario defined by the given boundary conditions specified by Tables 1 and 3; let us call it Scenario 1 .

Table 1 lists the given absolute fitness values for the sub populations.

\begin{tabular}{|l|l|}
\hline$W_{1}$ & 4.0 \\
\hline$W_{2}$ & 3.0 \\
\hline$W_{3}$ & 2.0 \\
\hline
\end{tabular}

Table 1: Absolute Fitness Values of Subpopulations (Scenario 1)

The given values in Table 1 result in the computed relative fitness values for Table 2:

\begin{tabular}{|c|c|}
\hline$w_{1}$ & 1.0 \\
\hline$w_{2}$ & 0.75 \\
\hline$w_{3}$ & 0.50 \\
\hline
\end{tabular}

Table 2: Relative Fitness Values (Scenario 1)

The given initial absolute individual/allele populations at generation 0 are listed in Table 3:

\begin{tabular}{|l|l|}
\hline$P_{1}(0)$ & 1 \\
\hline$P_{2}(0)$ & 2 \\
\hline$P_{3}(0)$ & 7 \\
\hline
\end{tabular}

Table 3: Absolute Individual/Allele Populations at Generation 0 (Scenario 1)

which results in the computed relative population frequencies in Table 4:

\begin{tabular}{|l|l|}
\hline$p_{1}(0)$ & 0.1 \\
\hline$p_{2}(0)$ & 0.2 \\
\hline$p_{3}(0)$ & 0.7 \\
\hline
\end{tabular}

Table 4: Relative Population Frequencies (Scenario 1)

Given the boundary conditions defined by Tables 1 and 3, the following values were computed over 5 generations for Scenario 1 and listed in Table 5 .

[Tables 1 through 5 were from given and computed values used in the associated supplementary spreadsheet, Supplement $1 .^{1}$ ]

One should readily observe that the last two columns of Table 5 have identical values! This is an illustration of Fisher's Fundamental Theorem of Natural selection in the simplest discrete generation, single locus, haploid model. The statement of the Fisher's theorem in the discrete generation model is:

$$
\Delta \overline{w(k)}=\frac{\operatorname{Var}(w(k))}{\overline{w(k)}}
$$

and is illustrated by the fact the last two columns are identical.

Running more generations, the mean fitness $\overline{w(k)}$ will approach 1.0, meaning the population will asymptotically approach homogeneity. Ironically a condition where all members of a population are all clones of each other might be deemed to actually be unhealthy since diversity of alleles in a population can help a species' survivability since an environmental change might eliminate some individuals carrying a certain allele but not others.

It must be emphasized (17) is not the most general form of Fisher's theorem despite the fact Barton and Queller refer to it as Fisher's theorem (Barton et al., 2007; Queller, 2017). Lessard and Ewens would call this the discrete generation case (Ewens and Lessard, 2015) for only one locus (albeit they use fairly different math symbols to describe the theorem). The most general statement Fisher's theorem was for the continuous generation case and for multiple loci (Price, 1972).

That said, below is the proof the discrete (17) version of Fisher's theorem for the haploid single locus case which follows almost exactly a proof provided in Joseph Felsenstein's graduate textbook, Evolutionary Theoretical Genet-

\footnotetext{
${ }^{1}$ Supplement 1 is available online at https://journals. blythinstitute.org/ojs/index.php/cbi/article/view/67/64.
} 


\begin{tabular}{|c|c|c|c|c|}
\hline generation $(k)$ & $\overline{w(k)}$ & $\operatorname{Var}(w(k))$ & $\Delta \overline{w(k)}$ & $\frac{\operatorname{Var}(w(k))}{\overline{w(k)}}$ \\
\hline 0 & 0.6000000 & 0.0275000 & 0.0458333 & 0.0458333 \\
\hline 1 & 0.6458333 & 0.0360243 & 0.0557796 & 0.0557796 \\
\hline 2 & 0.7016129 & 0.0420135 & 0.0598813 & 0.0598813 \\
\hline 3 & 0.7614943 & 0.0429713 & 0.0564303 & 0.0564303 \\
\hline 4 & 0.8179245 & 0.0387825 & 0.0474157 & 0.0474157 \\
\hline 5 & 0.8653403 & 0.0316793 & 0.0366091 & 0.0366091 \\
\hline
\end{tabular}

Table 5: Scenario 1 (5 Generations)

ics (Felsenstein, 2017), but with some clarifying notation added.

\subsection{Proof of (17)}

Starting from (10), the following must also hold by simple substitution of $k+1$ for $k$ :

$$
P_{i}(k+1)=P_{i}(0) W_{i}^{k+1}
$$

Thus, in light of (10) and (18):

$$
P_{i}(k+1)=P_{i}(0) W_{i}^{k+1}=P_{i}(0) W_{i}^{k} W_{i}=P_{i}(k) W_{i}
$$

Using this result we can say by extending (11) and (12):

$$
p_{i}(k+1)=\frac{P_{i}(k+1)}{P_{\text {tot }}(k+1)}=\frac{P_{i}(k+1)}{\sum_{i=1}^{N} P_{i}(k+1)}
$$

However, in light of (19), (20) can be restated as:

$$
p_{i}(k+1)=\frac{P_{i}(k+1)}{\sum_{i=1}^{N} P_{i}(k+1)}=\frac{W_{i} P_{i}(k)}{\sum_{i=1}^{N} W_{i} P_{i}(k)}
$$

We can simultaneously divide the numerator and denominator of (21) by $P_{\text {tot }}(k)$, and given (12), instead of relating $p_{i}(k+1)$ in terms of the absolute sizes $P_{i}(\mathrm{k})$ of the $i$ sub populations, we can express it in terms of the frequency of individuals (or alleles) $p_{i}$ in total population:

$$
p_{i}(k+1)=\frac{W_{i} p_{i}(k)}{\sum_{i=1}^{N} W_{i} p_{i}(k)}
$$

Referring back to equation (13), if we divide the numerator and denominator of (22) simultaneously by $W_{\text {ref }}$, we scale the absolute fitnesses down to relative fitnesses:

$$
p_{i}(k+1)=\frac{w_{i} p_{i}(k)}{\sum_{i=1}^{N} w_{i} p_{i}(k)}
$$

The term in the denominator is equal to the mean of the relative fitnesses $\overline{w(k)}$, thus

$$
p_{i}(k+1)=\frac{w_{i} p_{i}(k)}{\overline{w(k)}}
$$

By way of extension, equation (14) must hold true also for using $k+1$ :

$$
\overline{w(k+1)}=\sum_{i=1}^{N} p_{i}(k+1) w_{i}
$$

Substituting the righthand side of $(24)$ for $p_{i}(k+1)$ into (25) yields

$$
\overline{w(k+1)}=\sum_{i=1}^{N} \frac{w_{i} p_{i}(k)}{\overline{w(k)}} w_{i}=\sum_{i=1}^{N} \frac{p_{i}(k) w_{i}^{2}}{\overline{w(k)}}
$$

Subtracting $\overline{w(k)}$ from the right hand side and left hand side:

$$
\overline{w(k+1)}-\overline{w(k)}=\left\{\sum_{i=1}^{N} \frac{p_{i}(k) w_{i}^{2}}{\overline{w(k)}}\right\}-\overline{w(k)}
$$

Since

$$
\overline{w(k)}=\frac{\overline{w(k)}^{2}}{\overline{w(k)}}
$$


We can state (27) alternatively as:

$$
\overline{w(k+1)}-\overline{w(k)}=\frac{\left\{\sum_{i=1}^{N} p_{i}(k) w_{i}^{2}\right\}-\overline{w(k)}^{2}}{\overline{w(k)}}
$$

The numerator of (29) is the same as the variance $\operatorname{Var}(w(k))$ from $(15)$, thus

$$
\overline{w(k+1)}-\overline{w(k)}=\frac{\operatorname{Var}(w(k))}{\overline{w(k)}}
$$

noting equation (16) we have

$$
\Delta \overline{w(k)}=\frac{\operatorname{Var}(w(k))}{\overline{w(k)}}
$$

this completes the proof of the discrete-generation, haploid, one-locus case of Fisher's theorem.

It is worth mentioning many, if not all, texts will not use the notation here, especially the explicit statement of a variable's dependence on generation $k$. But the dependence on $k$ is emphasized here for clarity.

\subsection{How Much Difference Does it Make?}

Now suppose we have the following scenario defined by the boundary conditions described by Tables 6 and 7; let us call it Scenario 2. The absolute fitness and absolute population numbers need not be stated explicitly for Scenario 2 because (23) enables the computation of values for Table 8 with only relative fitnesses and the initial allele/population frequencies. One can simply assume there exists some absolute fitness values and initial absolute population sizes that will yield Tables 6 and 7 .

The relative fitness values which are constant for every generation are listed in Table 6:

\begin{tabular}{|l|l|}
\hline$w_{1}$ & 1.00000 \\
\hline$w_{2}$ & 0.53125 \\
\hline$w_{3}$ & 0.53125 \\
\hline
\end{tabular}

Table 6: Relative Fitness Values (Scenario 2)

The initial allele frequencies at generation 0 are listed in Table 7:

\begin{tabular}{|l|l|}
\hline$p_{1}(0)$ & 0.146666666 \\
\hline$p_{2}(0)$ & 0.426666667 \\
\hline$p_{3}(0)$ & 0.426666667 \\
\hline
\end{tabular}

Table 7: Initial Allele Frequencies (Scenario 2)

Given the boundary conditions defined by Tables 6 and 7 , the following values were computed over 5 generations for Scenario 2 and listed in Table 8.

[Tables 6 through 8 were from given and computed values used in the associated supplementary spreadsheet (Supplement 2$).^{2}$ ]

What is noteworthy is that for generation 0 , the entries for both Scenario 1 and Scenario 2 are identical! However, at generation 5, the mean fitness in Scenario 1 is 0.8653403 whereas for Scenario 2 it is 0.9073945 . This illustrates the obvious fact that different sets of relative fitness values $w_{i}$ can possibly result in identical mean fitness and variance values for some generations but not others. This shows that Fisher's theorem, in and of itself, cannot predict the trajectory of population structure. This is owing to the simple fact that infinite sets of numbers can share the same mean and variance somewhat analogous to how several different curves with different trajectories can share a point in a $2 \mathrm{D}$ plane.

Ewens and Lessard highlighted this fact as a part of a generally negative assessment of Fisher's theorem (Ewens and Lessard, 2015). It shows Fisher's theorem adds no greater insight to population trajectory than provided by the relative fitness values $w_{i}$ themselves and the relative initial allele frequencies $p_{i}(0)$. Thus, Fisher's Fundamental Theorem of Natural Selection adds no additional insight into the evolution of complexity, not only for the reasons stated by Ewens and Lessard, but also due to the definition of fitness used, namely fitness defined in terms of population growth rather than biophysical complexity.

\section{Discussion}

The difficulty of establishing the relevance Fisher's theorem to real-world questions about the evolution of complexity should be apparent because Fisher's theorem could just as well be applied to the increase of fitness due to loss of complexity. The numerical illustrations above have no indication whether the fitness values are due to gain or loss of complexity. It would appear Dawkins lionizing and mythol-

\footnotetext{
${ }^{2}$ Supplement 2 is available online at https://journals. blythinstitute.org/ojs/index.php/cbi/article/view/67/65.
} 


\begin{tabular}{|c|c|c|c|c|}
\hline generation $(k)$ & $\overline{w(k)}$ & $\operatorname{Var}(w(k))$ & $\Delta \overline{w(k)}$ & $\frac{\operatorname{Var}(w(k))}{\overline{w(k)}}$ \\
\hline 0 & 0.6000000 & 0.0275000 & 0.0458333 & 0.0458333 \\
\hline 1 & 0.6458333 & 0.0405816 & 0.0628360 & 0.0628360 \\
\hline 2 & 0.7086694 & 0.0516877 & 0.0729363 & 0.0729363 \\
\hline 3 & 0.7816056 & 0.0546763 & 0.0699538 & 0.0699538 \\
\hline 4 & 0.8515594 & 0.0475469 & 0.0558351 & 0.0558351 \\
\hline 5 & 0.9073945 & 0.0348330 & 0.0383880 & 0.0383880 \\
\hline
\end{tabular}

Table 8: Scenario 2 (5 Generations)

ogizing of Fisher's theorem is exactly that, since Fisher's theorem doesn't directly establish that biological complexity must necessarily increase.

The above discrete-generation version of Fisher's theorem can be generalized to a continuous multi-locus diploid version of Fisher's theorem. Fisher described his theorem for the continuous case where the continuous case does not require dividing $\operatorname{Var}(w(k))$ by $\overline{w(k)}$ in relating "rate of increase in fitness" to "genetic variance in fitness." The proof of the most general version is substantially more difficult, but was demonstrated by George Price in 1972 (Price, 1972), and thus not repeated here.

An amusing historical anecdote regarding Fisher's theorem is that he did not use sufficient rigor to describe his own fundamental theorem. For decades people accepted FTNS as true even though they did not understand it. It could be speculated this perpetuated its mythical status as biology's central theorem, even though population geneticists rarely, if at all, used FTNS after Fisher first introduced it. He stated his theorem in this way without rigorously defining the terms mathematically:

"The rate of increase in fitness of any organism at any time is equal to its genetic variance in fitness at that time."

(Fisher, 1930, pgs. 36-37)

These vague words lead George Price to observe in 1972:

It has long been a mystery how Fisher $(1930,1941$, 1958) derived his famous 'fundamental theorem of Natural Selection' and exactly what he meant by it... Also, he spoke of the 'rigour' of his derivation of the theorem and of 'the ease of its interpretation'. But others have variously described his derivation as 'recondite' (Crow \& Kimura, 1970), 'very difficult' (Turner, 1970), or 'entirely obscure' (Kempthorne, 1957). And no one has ever found any other way to derive the result that Fisher seems to state. Hence, many authors (not reviewed here) have maintained that the theorem holds only under very special conditions, while only a few (eg. Edwards, 1967) have thought that Fisher may have been correct - if only we could understand what he meant!

(Price, 1972)

However, as with the discrete-generation version, the most general version of Fisher's theorem suffers the same problems in terms of its lack of adding any further insight to the population's trajectory than already specified by the requisite boundary conditions. As with the discrete single locus haploid case, the most generalized FTNS adds no insight to the details of the evolution of biological systems in terms of the common sense notions of fitness used in the medical and engineering disciplines.

Amazingly, the original generalization of FTNS clarified by Price does not include effects of the infusion of additional mutations over time. Basener and Sanford demonstrated that given enough mutations, mean fitness will never maximize (Basener and Sanford, 2018).

Further, apart from even adding mutations, Patrick Moran and Richard Lewontin demonstrated that fitness is not guaranteed to maximize if epistasis and gene linkage are modeled. Grodwohl characterized these shocking mathematical developments as "The Rise and Fall of Fitness Maximization" (Grodwohl, 2016).

In light of the fact that most "beneficial" mutations are loss of function, then the individual with the highest fitness could be the one that has lost function, rather than gained it. This suggests that even in the existing framework of population genetics, natural selection could just as well eliminate function out of a population rather than construct it.

Thus, Fisher's theorem could just as well be used in models 
where natural selection facilitates destruction of function, and such destruction of function has direct observational confirmation in the field and in experiments as the overwhelming cause of beneficial mutations (Behe, 2010).

It is also worth mentioning that Ronald Brady, J. G. Ollason, and others point out the tautologous and inadequate definition of fitness in evolutionary biology, such that it has no utility in helping determine the veracity of evolutionary theory (Brady, 1979; Ollason, 1991).

Dawkins' Weasel program (Dawkins, 1986, pgs. 46-51) metaphorically describes how evolution is conceived in the minds of some and has been augmented and extended to teach evolution as part of an outreach program (Christensen-Dalsgaard and Kanneworff, 2009). Independent of the scholarly value of Dawkins' Weasel, metaphorically speaking an anti-Weasel model of evolution by natural selection might be a more valid description of how certain, if not most, organisms evolve by natural selection.

\section{Conclusion}

Fisher may have envisioned that his Fundamental Theorem of Natural Selection was proof that evolutionary progress is inevitable. However, Fisher's theorem is perfectly consistent with the view that reductive evolution is the dominant mode of evolution. And if direct observation of the ubiquity of reductive evolution can be extrapolated to the past, then complexity decrease by natural selection would be the norm, and thus complexity increase from simple bacteria to complex multicellular eukaryotes must come about by a mechanism other than natural selection. An anti-Weasel model of natural selection might more accurately describe the operation of natural selection in the wild.

Beyond that, on many levels Fisher's theorem has been given a negative assessment by Ewens and Lessard, was criticized by Price for not being understandable and to be incompletely derived and proven, and declared by Felsentein as being not-so-fundamental afterall. All of these considerations are in stark contrast to Dawkins' characterization of Fisher's theorem as biology's central theorem.

\section{References}

Adami, C, C Ofria, and T C Collier (2000). "Evolution of Biological Complexity". In: Proceedings of the $\mathrm{Na}$ - tional Academy of Science 97.9, pp. 4463-4468. DOI: 10.1073/pnas.97.9.4463.

Angier, N (2010). "Seeing the Natural World with a Physicist's Lens". In: The New York Times November 1, 2010.

Barton, N H et al. (2007). Evolution. Cold Spring Harbor: Cold Spring Harbor Press.

Basener, W F and J C Sanford (2018). "The Fundamental Theorem of Natural Selection with Mutations". In: Journal of Mathematical Biology 76, pp. 1589-1622. DOI: $10.1007 / \mathrm{s} 00285-017-1190-\mathrm{x}$.

Behe, M J (1996). Darwin's Black Box. New York: Touchstone, pp. 39-40.

Behe, M J (2010). "Experimental Evolution, Loss-OfFunction Mutations, and "The First Rule of Adaptive Evolution"". In: The Quarterly Review of Biology 85.4, pp. 419-445. DOI: $10.1086 / 656902$.

Bialek, W (2012). Biophysics: Searching for Principles. Princeton, NJ: Princeton University Press.

Bialek, W (2015). More Perfect Than We Imagined: A Physicist's View of Life. URL: https://www. cornell. edu/video/william-bialek-physicists-view-oflife.

Brady, R H (1979). "Natural Selection and the Criteria by Which a Theory is Judged". In: Systematic Biology 28.4, pp. 600-621. DOI: $10.2307 /$ sysbio/28.4.600.

Brockman, J (2011). "Who is the Greatest Biologist of All Time?" In: URL: https : / / www . edge . org / conversation/armand_marie_leroi-who-is-thegreatest-biologist-of-all-time.

Christensen-Dalsgaard, J and M Kanneworff (2009). "Evolution in Lego: A Physical Simulation by Natural Selection". In: Evolution: Education and Outreach 2, pp. 518-526. DOI: $10.1007 / \mathrm{s} 12052-008-0099-7$.

Darwin, C (1859). "Organs of Extreme Perfection and Complication". In: On the Origin of Species by Means of Natural Selection, or the Preservation of Favoured Races in the Struggle for Life.

Dawkins, R (1986). The Blind Watchmaker. Oxford University Press.

Ewens, W J and S Lessard (2015). "On the interpretation and relevance of the Fundamental Theorem of Fundamental Theorm of Natural Selection". In: Theoretical Population Biology 104, pp. 59-67. DOI: $10.1016 / \mathrm{j}$. tpb.2015.07.002.

Felsenstein, J (2017). Theoretical Evolutionary Genetics. Seattle, WA. URL: http : / / evolution - genetics . washington.edu/pgbook/pgbook.html.

Felsenstein, J (2018). Fisher Memorial Lecture 2018. URL: https://www. youtube. com/watch?v=ZF3nIMvBBDw.

Fisher, R A (1930). "The Genetical Theory of Natural Selection". In: DOI: 10.5962/bhl.title. 27468. 
Grodwohl, J B (2016). "“The Theory was Beautiful Indeed": Rise, Fall, and Circulation of Maximizing Methods in Population Genetics (1930-1980)". In: Journal of the History of Biology 50.3, pp. 571-608. DOI: 10 . 1007/s10739-016-9449-4.

Lewontin, R (2003). "Four Complications in Understanding the Evolutionary Process". In: Santa Fe Institute Bulletin 18.1. URL: https://sfi-edu.s3.amazonaws. com/sfi-edu/production/uploads / publication / 2016/10/31/winter2003v18n1.pdf.

Moalem, S (2008). Survival of the Sickest. New York: Harper Perennial.

Ollason, J G (1991). "What is this Stuff Called Fitness?" In: Biology and Philosophy 6, pp. 81-92. DOI: 10.1007/ BF02426827.

Orr, H A (1996). "Dennett's Strange Idea". In: Boston Review 21.3. URL: https : / / bostonreview . net / archives/BR21.3/Orr.html.

Orr, H A (2009). "Fitness and Its Role in Evolutionary Genetics". In: Nature Reviews Genetics 10, pp. 531539. DOI: $10.1038 / \mathrm{nrg} 2603$.

Price, G R (1972). "Fisher's 'Fundamental Theorem' Made Clear". In: Annals of Human Genetics 36.2, pp. 129140. DOI: 10.1111/j.1469-1809.1972.tb00764.x.

Queller, D C (2017). "Fundamental Theorems of Evolution". In: The American Naturalist 189.4, pp. 345-353. DOI: $10.1086 / 690937$.

Ritz, T, S Adem, and K Schulten (2000). "A Model for Photoreceptor-based Magnetoreception in Birds". In: Biophysical Journal 78.2, pp. 707-718. DOI: 10.1016/ S0006-3495(00) 76629-X.

Royal Society (2020). "Ronald Fisher". In: URL: https : //makingscience.royalsociety.org/s/rs/people/ fst00034451.

Rubenstein, D I and M A Hack (2013). "Migration". In: Encyclopedia of Biodiversity. Ed. by S A Levin. Second Edition. Elsevier, pp. 309-320.

Vaughan, N (2019). "Evolution of Biological Eye in Computer Simulation". In: 2019 IEEE Congress on Evolutionary Computation (CEC). DOI: 10.1109/CEC. 2019. 8790284.

Wagner, A (2005). Robustness and Evolvability in Living Systems. New Jersey: Princeton University Press.

Wolf, Y I and E V Koonin (2013). "Genome Reduction as the Dominant Mode of Evolution". In: Bioessays 35.9, pp. 829-837. DOI: 10.1002/bies. 201300037. 